\title{
Pressurization of a Flightweight, Liquid Hydrogen Tank: Evaporation \& Condensation at a Liquid/Vapor Interface
}

\author{
Mark E. M. Stewart ${ }^{1}$ \\ VPL LLC at NASA Glenn Research Center, Cleveland, Ohio, 44135, USA
}

\begin{abstract}
This paper presents an analysis and simulation of evaporation and condensation at a motionless liquid/vapor interface. A 1-D model equation, emphasizing heat and mass transfer at the interface, is solved in two ways, and incorporated into a subgrid interface model within a CFD simulation. Simulation predictions are compared with experimental data from the CPST Engineering Design Unit tank, a cryogenic fluid management test tank in 1-g. The numerical challenge here is the physics of the liquid/vapor interface; pressurizing the ullage heats it by several degrees, and sets up an interfacial temperature gradient that transfers heat to the liquid phase-the rate limiting step of condensation is heat conducted through the liquid and vapor. This physics occurs in thin thermal layers $O(1$ $\mathbf{m m}$ ) on either side of the interface which is resolved by the subgrid interface model. An accommodation coefficient of 1.0 is used in the simulations which is consistent with theory and measurements. This model is predictive of evaporation/condensation rates, that is, there is no parameter tuning.
\end{abstract}

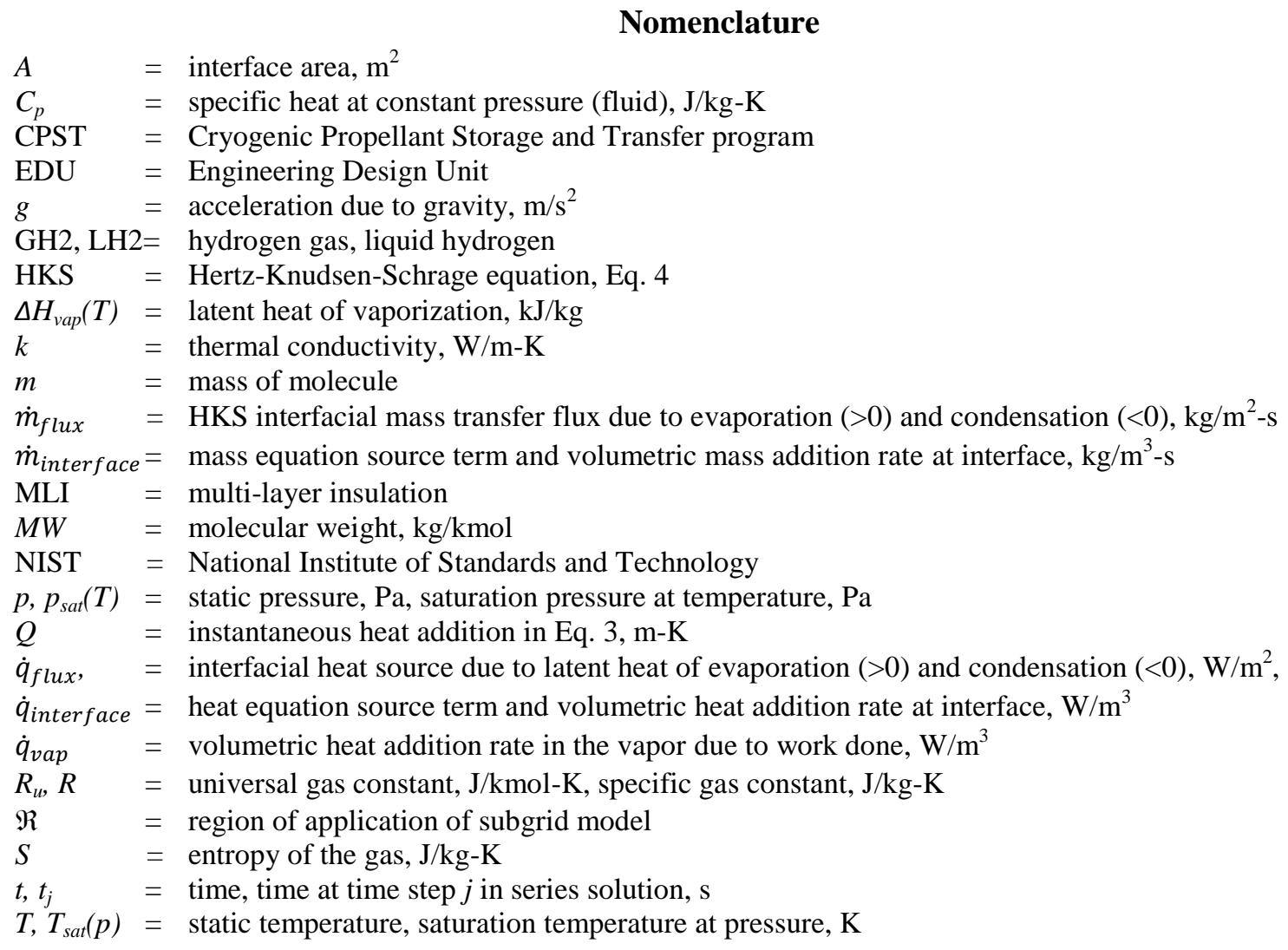

${ }^{1}$ Senior Research Engineer, MS VPL-3, AIAA Member. 
$T_{\text {interface }}=$ temperature of the liquid/vapor interface, $\mathrm{K}$

$\Delta T_{\text {compress }}=$ temperature change of a gas due to isentropic compression/expansion between $1 \& 2 \mathrm{~atm}, \mathrm{~K}$

$u, v, w=$ velocity components for molecules, $\mathrm{m} / \mathrm{s}$

$\mathrm{UDF} \quad=$ user defined function (ANSYS Fluent)

$V \quad=$ volume, $\mathrm{m}^{3}$

VOF $=$ volume of fluid numerical method

$W \quad=$ work, done on a compressible gas, $\mathrm{J}$

$x \quad=$ coordinate normal to interface, $\mathrm{m}$

$\alpha=$ heat equation parameter characterizes sharpness/persistence of temperature gradient, $\mathrm{m}^{2} / \mathrm{s}$

$\beta, \chi=$ subgrid model mass and energy scaling factors between flux and equation source terms, unitless

$\gamma \quad=$ ratio of specific heats, unitless

$\rho \quad=$ density, $\mathrm{kg} / \mathrm{m}^{3}$

$\Sigma_{\Re} \quad=$ summation over cells in region $\Re$

$\sigma_{\text {cond }}, \sigma_{\text {evap }}=$ mass accommodation coefficients for condensation and evaporation, unitless, $[0,1]$

$\varphi_{\text {liq }}, \varphi_{\text {vap }}=$ liquid, vapor fraction at a point, unitless, $[0,1]$

eff $\quad=$ source term actually added to equation in numerical scheme

interface = subscript indicating the liquid/vapor interface

liq, vap, solid = subscripts indicating liquid, vapor, and solid phases

sat $\quad=$ saturation conditions

\section{Introduction}

$\mathrm{N}$ ASA is interested in long-duration, in-space storage of cryogenic propellants to support future exploration missions, including upper stages and potentially propellant depots. Cryogenic propellants promise higher specific impulse than storable hypergolic fuels, but storage for long duration missions has yet to be demonstrated. Improving the capabilities of computational tools to predict fluid dynamic and thermodynamic behavior in cryogenic propellant tanks under settled and unsettled conditions is research supported under NASA's Evolvable Cryogenics (eCryo) project.

A challenging problem for these multiphase computational methods is predicting mass transfer (evaporation and condensation) at the liquid/vapor interface; the symptoms include poor prediction of pressure in some cases. A typical approach is to combine an interface tracking method (which captures interface position and motion) with a mass transfer equation to predict evaporation/condensation [1] [2]. Typically, the interface is smeared over several grid cells, hence smaller length scales are ignored near the interface.

This problem is challenging because it involves at least three problems, each from different disciplines: the statistical kinetics of evaporation and condensation at the phase interface, the heat transfer in the adjacent phases, and numerical methods must capture all this physics.

In this paper, heat transfer near the interface is demonstrated as a rate limiting step for evaporation/condensation at the interface-particularly when the fluids have poor thermal conductivity. For small pressure changes, the Hertz-Knudsen-Schrage equation predicts high mass transfer rates and huge heat generation/absorption rates. But, in many situations, the adjacent vapor and liquid cannot conduct this heat. The result is thin, $\mathrm{O}(1 \mathrm{~mm})$, thermal layers on both sides of the interface. We would not be surprised at a thermal boundary layer at a wall; liquid/vapor interfaces can have a pair of thermal layers with a heat source/sink in between!

This behavior is apparent in the engineering problem of interest here: pressurization of a liquid hydrogen tank. The

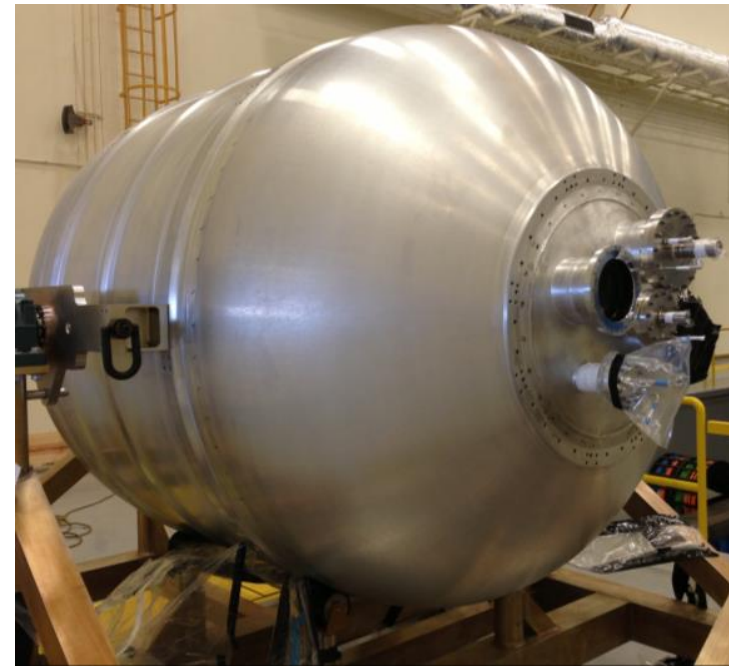

Figure 1: CPST EDU tank during fabrication at Marshall Space Flight Center. External view of tank without MLI or foam. EDU (Engineering Design Unit), Figure 1, is a cryogenic fluid management test article developed as part of the CPST program, a precursor to the eCryo program. Tests at Marshall Space Flight Center in 2014 and 2015 included 
pressurizing liquid cryogens (principally hydrogen) with various gases using unsubmerged and submerged diffusers, plus tank drainage with pressure control, all in 1-g.

This interfacial behavior is investigated through both exact and numerical solutions for heat and mass transfer near the liquid/vapor interface. Section II shows how interfacial temperature jumps can occur through (de)compression, while Section III discusses the liquid/vapor interface and evaporation/condensation. Section IV establishes a model of heat conduction and thermal layers using the one-dimensional heat equation through the interface and nearby vapor/liquid. Two different solution methods provide validation. Section V explains a numerical method where the heat equation model, plus solution method, is used as a subgrid model within an ANSYS Fluent simulation. Section VI explains the engineering experiment, geometry and the grid used. Section VII compares Fluent simulation results with EDU Tank pressurization and drainage experimental results.

\section{Temperature Jumps at the Interface: When Thermal Gradients Become Sharp}

Interfacial temperature jumps (large gradients) can occur for a simple reason: pressurization of the vapor phase does work, $W$, on the gas (Eq. 1), and raises its temperature everywhere-including up to the interface [3, p. 6]; the incompressible fluid has no volume change or work done on it. The resulting temperature gradient sets up a heat flow across the interface, as shown in Figure 2. The opposite holds true; depressurization will lower the gas

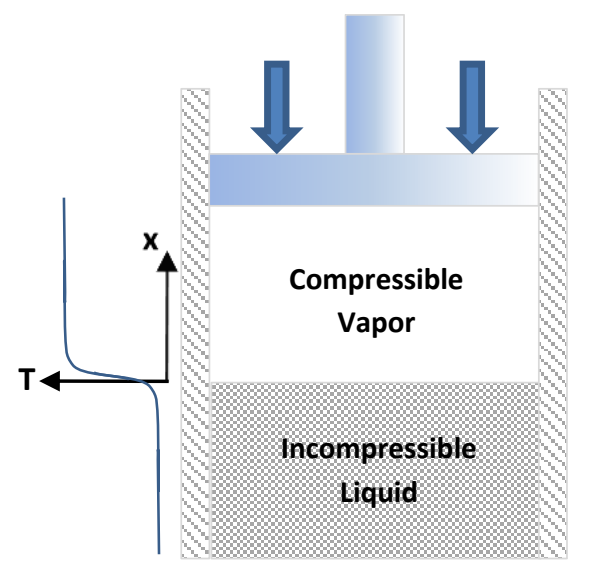

Figure 2: Pressurization of gas leads to a temperature jump. Work is done on the compressible vapor phase which raises its temperature-right up to the interface. No compression or work is done on the incompressible liquid phase. Hence pressurization leads to a temperature jump. Depressurization works in the opposite manner. 'Incompressible Liquid' is used in the fluid mechanics sense-negligible compression. Most liquids undergo small compression compared to gases. temperature and create the reverse gradient. Pop the cap off a soft drink, and the pressure drop yields a temperature drop in the vapor phase.

$$
W=-\int p d V
$$

Temperature increases for isentropic compression, $\Delta T_{\text {compress }}$, from 1 to 2 atmospheres are shown in Table 1 for several gases; hydrogen has a modest temperature jump of $4.44 \mathrm{~K}$, while water is $70 \mathrm{~K}$ between 1 and 2 atm.

The persistence in time of this temperature jump is important, and depends on how quickly heat is conducted. This transient thermal conductivity is captured in the one-dimensional heat equation, Eq. 2, where $\dot{q}_{v a p}$ represents the work done on the vapor by compression/decompression. Section IV-A shows how a series of exact solutions, Eq. 3, give the time development of the temperature gradient, plus its thickness. Section IV-B explains a numerical solution method.

$$
\frac{\partial T}{\partial t}-\alpha \frac{\partial^{2} T}{\partial x^{2}}=\frac{1}{\rho C_{p}} \dot{q}_{v a p} \quad \text { where } \alpha=\frac{k}{C_{p} \rho}
$$

Table 1 gives values of $\alpha$ for common gases, and smaller $\alpha$ values indicate sharper, more persistent temperature gradients.

$$
T(x, t)=T_{\infty}+\frac{Q}{(4 \pi \alpha t)^{1 / 2}} e^{-\frac{x^{2}}{4 \alpha t}}
$$

where $\rho C_{p} Q$ is the heat per unit area added instantaneously at $x=$ $t=0$.

Figure 3 demonstrates the persistence of a temperature jump in hydrogen liquid/vapor after aggressive pressurization in the first 10 seconds. In the minute after pressurization, the width of the pressure jump grows from a few millimeters to several centimeters. After several minutes, the temperature profile approaches the constant slope, steady-state profile.

With slow vapor pressurization, temperature jumps are virtually non-existent. Stewart [2] shows that the small, constant-slope temperature gradients of self-pressurization can be captured with a fine grid at a fixed interface.

Figure 2's piston, as in an internal combustion engine, is only one method of compression/decompression and one cause of interfacial temperature gradients. Compression — and increasing gas temperature-occurs similarly in compressors within jet engines and air conditioners, boiling within a closed tank [4], change in altitude [5], or flowing gas through a diffuser into a propellant tank as considered in the EDU tank in Section VI. Interfacial temperature gradients are also caused by heat flows within a tank [2]. 
Table 1: Condensation/evaporation properties of common fluids at 1 atmosphere saturated conditions. NIST data [7]. $\Delta T_{\text {compress }}$ is isentropic.

\begin{tabular}{|c|c|c|c|c|c|c|c|}
\hline & $\begin{array}{c}\text { Latent Heat } \\
\Delta H_{\text {vap }} \\
(\mathrm{J} / \mathrm{kg})\end{array}$ & $\begin{array}{l}T_{\text {sat }} \\
(\mathbf{K})\end{array}$ & $\begin{array}{c}\Delta T_{\text {compress }} \\
1 \rightarrow 2 \mathrm{~atm} \\
(\mathrm{~K})\end{array}$ & $\begin{array}{c}\text { Vapor } \\
\Delta H_{v a p} / C_{p} \\
(\mathbf{K})\end{array}$ & $\begin{array}{c}\text { Liquid } \\
\Delta H_{\text {vap }} / C_{p} \\
(\mathbf{K})\end{array}$ & Vapor $\alpha$ & Liquid $\alpha$ \\
\hline Helium & 20,752 & 4.2304 & 1.342 & 2.3 & 3.9 & $5.95 \mathrm{E}-05$ & $2.82 \mathrm{E}-05$ \\
\hline Parahydrogen & $445,440$. & 20.277 & 4.441 & 36.4 & 46.1 & $1.04 \mathrm{E}-03$ & $2.81 \mathrm{E}-06$ \\
\hline Nitrogen & $199,178$. & 77.355 & 16.942 & 177.2 & 97.6 & $1.45 \mathrm{E}-03$ & $8.86 \mathrm{E}-05$ \\
\hline Oxygen & 213,050 & 90.188 & 19.752 & 219.5 & 125.4 & $1.93 \mathrm{E}-03$ & $7.82 \mathrm{E}-05$ \\
\hline Methane & $510,830$. & 111.67 & 20.433 & 230.3 & 146.7 & $2.88 \mathrm{E}-03$ & $1.25 \mathrm{E}-04$ \\
\hline Water & $2,256,440$. & 373.12 & 70.019 & 1084.9 & 535.3 & $2.02 \mathrm{E}-02$ & $1.68 \mathrm{E}-04$ \\
\hline
\end{tabular}

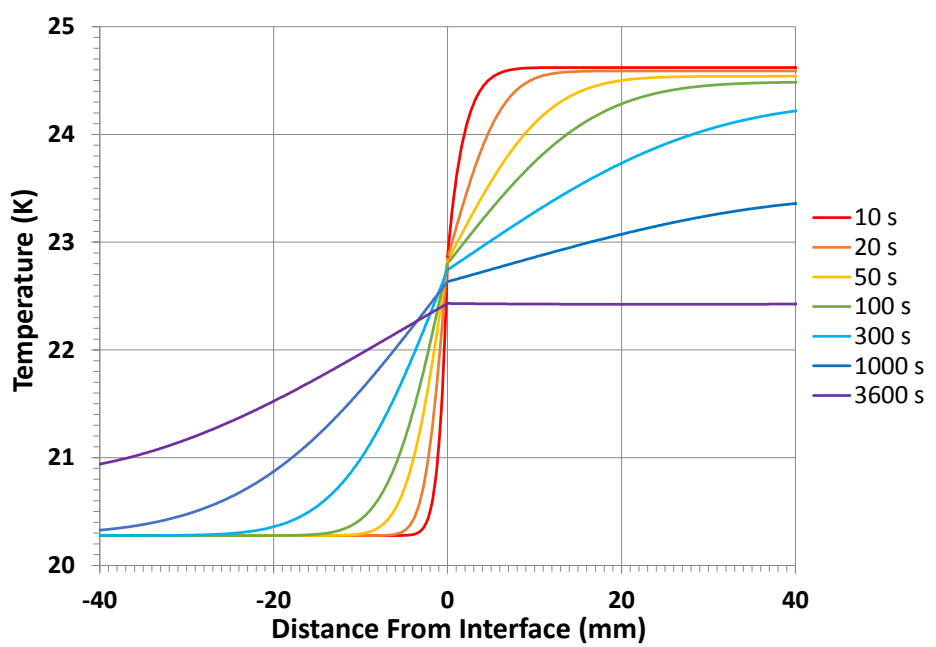

Figure 3: Temperature profiles through a hydrogen liquid/vapor interface at various times after pressurization from $P_{\text {sat }}(21 \mathrm{~K})=101,325 \mathrm{~Pa}$ to $202,650 \mathrm{~Pa}$ in 10 seconds. Includes interface condensation. Liquid for negative interface distances.

probe. Badam et al. [7] experimentally investigated temperature jumps due to a vapor side heat flux.

Heat transfer during condensation at a liquid/vapor interface is important in cloud formation, as the nucleation and growth of cloud droplets is relevant to critical climate change questions about cloud albedo. The Mason equation [5, p. 122] models growth of water droplets where diffusion of mass is balanced by diffusion of latent heat through the gas surrounding the droplet.

Evaporation and condensation can be relevant in acoustic problems. While measuring the sound speed of various gases using sound waves, investigators noted that for strong sound waves the pressure can exceed saturation pressure during part of the oscillation and be less during the remainder [10]. This leads to momentary, alternating condensation and evaporation. This complicated sound speed measurements. However, it provides an opportunity to measure evaporation/condensation where there is reduced net heat flow [11].

\section{Physics of the Fluid/Vapor Interface, Mass and Energy Transfer}

The Knudsen or vapor layer, where gas behavior is dominated by interaction with the adjacent liquid (or solid), is thin - a few mean free path lengths thick, and much less than a micrometer, $\mu$ m. This Knudsen layer is much thinner than the adjacent thermal boundary layers, and the gas is locally not in equilibrium in any layer.

The Schrage equation [12, p. 27] (Hertz-Knudsen-Schrage equation) estimates the evaporation/condensation mass flux and is derived solely from statistical mechanics and the thermodynamic states of the liquid and vaporheat transfer is not considered. Temperatures and pressures are assumed to be continuous-everywhere; jumps are assumed to be steep gradients. Hence, in Eq. $4, T_{\text {liq }}=T_{\text {vap }}=T_{\text {interface, }}, p_{\text {vap }}=p_{\text {interface }}$.

\section{4}

American Institute of Aeronautics and Astronautics 


$$
\dot{m}_{\text {flux }}\left(p_{\text {interface }}, T_{\text {interface }}\right)=\frac{2}{2-\sigma_{\text {cond }}} \sqrt{\frac{M W}{2 \pi R_{u}}}\left(\sigma_{\text {evap }} \frac{p_{\text {sat }}\left(T_{\text {liq }}\right)}{\sqrt{T_{\text {liq }}}}-\sigma_{\text {cond }} \frac{p_{\text {vap }}}{\sqrt{T_{\text {vap }}}}\right)
$$

Mathematically for the HKS equation, $\sigma_{\text {evap }}=\sigma_{\text {cond }}$ must be true for equilibrium to be satisfied at $p_{\text {sat }}\left(T_{\text {interface }}\right)$.

Accompanying evaporation (condensation) at the interface is the absorption (release) of large amounts of heat, Eq. 5. Table 1 gives the heat of vaporization, $\Delta H_{v a p}\left(T_{s a t}\right)$, for some common fluids. Note that $\Delta H_{v a p} / C_{p}$ compares the heat of vaporization to specific heat (heat required to raise temperature) — and it's a large amount of heat, particularly for water! The corresponding mass transfer is small. In this work, heat release is assumed to occur within the Knudsen layer, while outside, heat conduction is satisfied.

\section{Background}

$$
\dot{q}_{\text {flux }}=\dot{m}_{\text {flux }}\left(p_{\text {interface }}, T_{\text {interface }}\right) \Delta H_{\text {vap }}\left(T_{\text {interface }}\right)
$$

Schrage [12] gives a good review of interface physics up to 1950. Considerable vaporization/condensation research has been done on liquid/solid interfaces. The Hertz-Knudsen equation gives the time rate of gas molecules sticking to a solid surface as the product of a "sticking" coefficient, $\sigma$, (mass accommodation coefficient) and the flux of gas molecules impinging on the surface, $\dot{m}_{\text {flux }}^{\text {vap-solid }}$.

Schrage extended this to evaporation/condensation at liquid/vapor interfaces as two competing fluxes, Eq. 6. Each mass flux, Eq. 7 [12], is the expected number of molecules (in a Maxwellian velocity distribution, $f$ ) impinging on the interface. Pressure is the corresponding integral for momentum, so it's not surprising that the mass flux is a function of gas pressure and temperature. The two fluxes balance at saturation conditions, so the net flux is the deviation from saturation conditions, Eq. 4.

$$
\begin{gathered}
\dot{m}_{\text {flux }}=\sigma_{\text {evap }} \dot{m}_{f l u x}^{l i q \rightarrow \text { vap }}-\sigma_{\text {cond }} \dot{m}_{\text {flux }}^{\text {vap } \rightarrow \text { liq }} \\
\dot{m}_{\text {flux }}^{\text {liq } \rightarrow \text { vap }}=\int_{-\infty}^{\infty} \int_{-\infty}^{\infty} \int_{0}^{\infty} m u f(p, T, \boldsymbol{u}) d u d v d w=\sqrt{\frac{M W}{2 \pi R_{u}}} \frac{p}{\sqrt{T}}
\end{gathered}
$$

The Maxwell distribution, $f$, is a normal distribution of molecular velocities, and, strictly speaking, it only applies to equilibrium conditions - temperature gradients are non-equilibrium. The remaining question is the value of the accommocation coefficients, $\sigma_{\text {evap }}$, $\sigma_{\text {cond }}-$ what fraction of impinging molecules actually complete the phase transfer and stick?

\section{Accommodation Coefficients:}

For sublimation of solids, a number of experiments indicate mass accommodation coefficient, $\sigma$, values near 1. [12, p. 30]. Early experiments measuring these coefficients for liquids were less successful [12, p. 40].

Modern computers and molecular dynamics simulations were not available to early investigators, and these methods go beyond a Maxwellian distribution and "sticking" coefficient to model Coulombic and other force interations at atoms and groups within molecules as their trajectories are followed. Molecular dynamics computer simulations of the air/water interface give mass and thermal accommodation coefficients of 0.99 and 1.0 , respectively, at $300 \mathrm{~K}[13]$. For methanol and argon, condensation is complete capture with total condensation, $\sigma$, $\sim 0.2$ [14] and $\sim 0.8[15]$ respectively.

Experimentally, the thermal accommodation coefficient was shown to be $0.84 \pm 0.05$ in argon at $271 \mathrm{~K}$ using sound resonance in a spherical chamber [11]. The experiment creates strong sound waves with a pressure oscillation both above and below saturation pressure-so evaporation and condensation alternate with the pressure wave; a liquid layer can exist on the walls but without a net heat flow [10].

\section{A Model for Interface Mass and Energy Transfer}

Any successful mathematical model represents the dominant physical processes, and includes the corresponding terms in the equations. Smaller effects, and their terms, can be excluded, with care. Geometry, length, and time scales can be important. The dominant terms in the heat equation, Eq. 8, are heating of the fluid, $\rho C_{p} \frac{\partial T}{\partial t}$, thermal conduction through each phase, $-k \frac{\partial^{2} T}{\partial x^{2}}$, the rate of work done on the gas as it (de)compresses, $\dot{q}_{v a p}$, and latent heat absorbed or released at the interface, $\dot{q}_{\text {interface. }}$. This equation can be derived from the Navier-Stokes Energy equation, when two processes are negligible: fluid motion and temperature variation in the plane of the interface. 


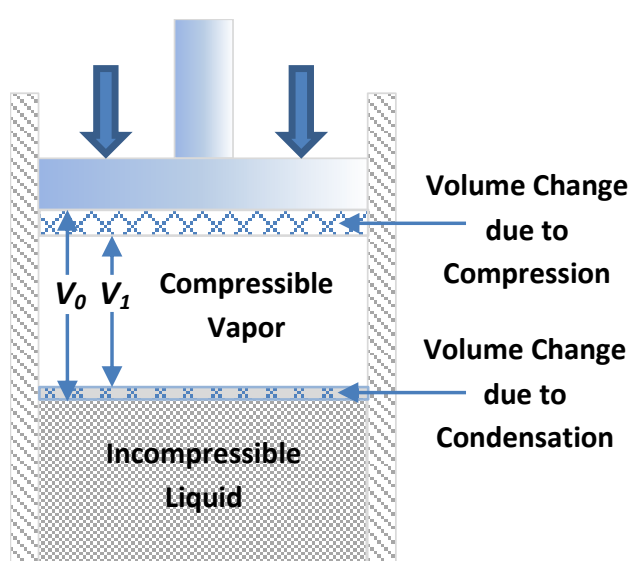

Figure 4: Mass is conserved and vapor temperature is predicted from gas volume change in Eq. 9. The heat equation is solved in a direction normal to the interface. Fluid motion and temperature variations in the interface plane are assumed to be negligible.

$$
\rho C_{p} \frac{\partial T}{\partial t}-k \frac{\partial^{2} T}{\partial x^{2}}=\dot{q}_{\text {interface }}+\dot{q}_{v a p}
$$

Note that fluid properties, $\rho, C_{p}, k$, switch between liquid and vapor at the interface.

The model conserves not only energy, but mass (Figure 4). The actual mass transfer at the interface, $\dot{m}_{\text {flux }}$ in Eq. 4 , is small, but included in each model through isentropic compression (an adiabatic reversible process for a perfect gas), Eq. 9 [3, pp. 14, 21] with $d S=0$.

$$
\frac{T_{1}}{T_{0}}=e^{\frac{d S}{C_{p}}}\left(\frac{V_{0}}{V_{1}}\right)^{\gamma-1}=e^{\frac{d S}{C_{p}}}\left(\frac{p_{1}}{p_{0}}\right)^{1-\frac{1}{\gamma}}
$$

\section{A. Exact Solutions for Time Evolution of Interfacial Temperature Gradients}

Analytic solutions to this equation, (8), are desirable because they give the interface profile, regardless of how thin the thermal layers are; with numerical solutions, trial and error and possibly a very fine grid are required - errors and uncertainty are possible.

The heat equation, Eq. 2, has an exact solution, Eq. 3, for instantaneous heat addition at $x=t=0$. A solution also exists for the two-phase heat equation, Eq. 8, with work done on the vapor and interfacial heat release-both time dependent. Temperature evolution can be predicted with series solutions, Eq. 10 and 11. Each solution in the series corresponds to instantaneous heat addition at a time $t_{j}$. Each phase has a separate solution matched at the interface with latent heat release.

$$
\begin{aligned}
& T^{v a p}(x, t)=T_{\infty}^{v a p}(t)+\sum_{j=1}^{t_{j} \leq t} \frac{Q_{j}^{v a p}}{\left(4 \pi \alpha_{v a p}\left(t-t_{j}\right)\right)^{1 / 2}} e^{\frac{-x^{2}}{4 \alpha_{v a p}\left(t-t_{j}\right)}}, \quad x \geq 0 \\
& T^{l i q}(x, t)=T_{-\infty}^{l i q} \quad+\sum_{j=1}^{t_{j} \leq t} \frac{Q_{j}^{l i q}}{\left(4 \pi \alpha_{l i q}\left(t-t_{j}\right)\right)^{1 / 2}} e^{\frac{-x^{2}}{4 \alpha_{l i q}\left(t-t_{j}\right)}}, \quad x \leq 0
\end{aligned}
$$

Liquid temperature far from the interface, $T_{-\infty}^{l i q}$, is assumed constant, and gas temperature far from the interface, $T_{\infty}^{v a p}(t)$, is calculated from isentropic compression, Eq. 9 and Figure 4, based on a specified compression rate.

Matching solutions at the interface is done carefully, as the HKS equation is very sensitive to pressure and temperature variations. At the interface, an iterative method is used to satisfy temperature continuity and energy conservation, Eq. 12, between the two series solutions at each time $t_{j}$.

$$
\left(-k_{\text {liq }} \frac{d T}{d x}\right)_{\text {interface-liq }}-\left(-k_{\text {vap }} \frac{d T}{d x}\right)_{\text {interface-vap }}=\dot{q}_{f l u x}=\dot{m}_{f l u x}\left(p_{\text {interface }}, T_{\text {interface }}\right) \Delta H_{\text {vap }}\left(T_{\text {interface }}\right)
$$

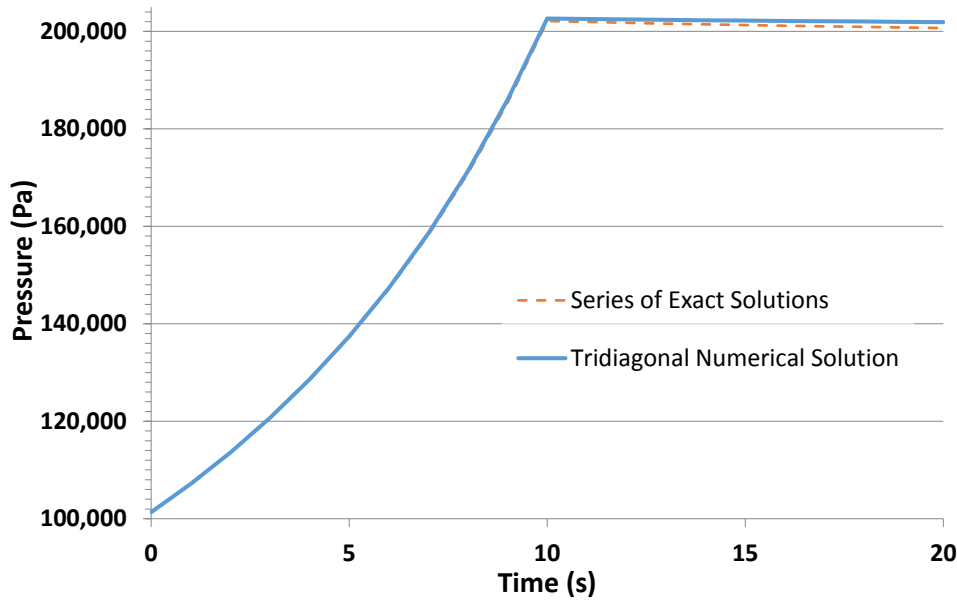

Figure 5: Pressure evolution for both solution techniques.

\section{B. Numerical Solutions for Time Evolution of Interfacial Temperature Gradients}

Eq. 8 can also be solved with a numerical method. In particular, a discrete, secondorder approximation to Eq. 8 can be solved with the Thomas algorithm for a tridiagonal matrix [16]. Typically, $10^{3}$ points with a uniform grid spacing of $\Delta \mathrm{x}=10^{-5} \mathrm{~m}$, time step of $\Delta \mathrm{t}=10^{-4} \mathrm{~s}$, are used.

Again, the interface conditions must be solved carefully. At the interface, a subiteration is used to find a consistent interface temperature, $T_{\text {interface }}$, and pressure, $p_{\text {interface, }}$, HKS mass flux, $\dot{m}_{\text {flux }}$, and heat generation rate, $\dot{q}_{f l u x}$, at each time step, Eq. 12. 
Far from the interface at the ends of the 1-D grid, boundary conditions are specified heat flux (temperature gradient). In this simulation, they are taken as zero.

Verification of numerical methods is enhanced when two different numerical methods can be applied to the same physical problem. In this case, results compare well as shown in Figure 5.

\section{Numerical Methods}

Physics can be forgiving of numerical methods, and sometimes it's not. Shocks can be smeared over several grid cells, and still yield good transonic lift predictions. Airfoil boundary layers can be ignored and still predict lift and drag due to lift (Euler equations). Yet try to predict heat transfer — on a turbine blade, for example - and hard work is required to get results within 10\%. Why? Pressure is constant across the boundary layer which is numerically trivial, while heat transfer depends on the derivative of the temperature profile through the boundary layer. Physics is forgiving in the lift calculation, but unforgiving in the heat transfer one.

Here, the detailed temperature profile cannot be smeared over large grid cells-much higher spatial resolution is required to resolve heat transfer, predict evaporation/condensation and pressure changes. Consequently, the numerical methods used here carefully resolve any temperature profiles at the liquid/vapor interface with a subgrid model.

\section{A. Numerical Scheme}

ANSYS Fluent version 16.0 [17] is used to solve thermal equations in the solid walls coupled to thermal/fluid equations in the fluid region (two-dimensional, axisymmetric, Navier-Stokes equations). The fluid flow is modeled as laminar flow. This transient simulation is second-order in space and time. The time step is 0.001 to $0.0005 \mathrm{~s}$.

Typically, in multiphase simulations, the VOF method [18] has a single, combined energy equation for both phases of the fluid. Consequently, heat exchange between the phases is adding/subtracting latent heat, yet sharp temperature gradients must be captured and resolved on a coarse grid. Here, two energy equations are used in the fluid equations, one for each phase - plus the subgrid model's heat equation gives fine grid resolution for the sharp gradients. In a single energy equation, numerical dissipation could smear the energy of the two phases - additional numerical heat transfer. In ANSYS Fluent, multiple energy equations to clearly separate the energy of multiple phases is Eulerian Multiphase [18]. This calculation uses the implicit Multifluid VOF sub-option.

\section{B. UDFs for MLI, Pressurant, Tank Venting and Drainage}

A Fluent simulation can be modified by including User Defined Functions (UDFs). This simulation makes extensive use of these subroutines. The tank exterior boundary condition includes the heat transfer effects of insulating foam, MLI, and radiation, all coded in a UDF [2]. Pressurizing gas (hydrogen) is introduced into the tank through a pipe and diffuser; the inflow boundary condition is a UDF which prescribes mass flow according to a schedule. To maintain a fixed pressure during tank settling, a venting UDF removes mass, momentum, and energy from a small region of the tank. A similar UDF allows for tank drainage.

The subgrid model uses UDFs to include source/sink terms in the mass, momentum, and liquid and vapor energy equations.

\section{A Subgrid Model}

To capture the thermal layers of the liquid/vapor interface when evaporation and condensation are present, a subgrid model is used. In theory, a fine grid could resolve the layers. In practice, the interface can move and curve; pressure increases and temperature gradients can be large. Consequently, the grid generation issues alone would be substantial, even for an unstructured, adaptive grid.

The approach here is to use a subgrid model that can change position on the grid, so the model remains at the liquid/vapor interface even as it moves. The underlying Fluent grid remains unchanged. The subgrid model solves the HKS equation and the 1-D heat equation, Eq. 8, normal to the interface using a tridiagonal matrix scheme, as in Section IV-B. This heat equation's grid floats at the moving position ( $\left.x_{\text {interface }}, y_{\text {interface }}\right)$, as shown in Figure 6 . It approximates the interface within a larger region, denoted $\mathfrak{R}$. The subgrid model consists of several UDFs coupling the 1-D heat equation and the Fluent calculations.

\section{Couplings between subgrid model and fluid simulation}

There are four couplings between the subgrid model and the fluid simulation. First, from the fluid simulation to the subgrid model, interface position calculated in the fluid simulation must update the position in the subgrid model, Eq. 13. 


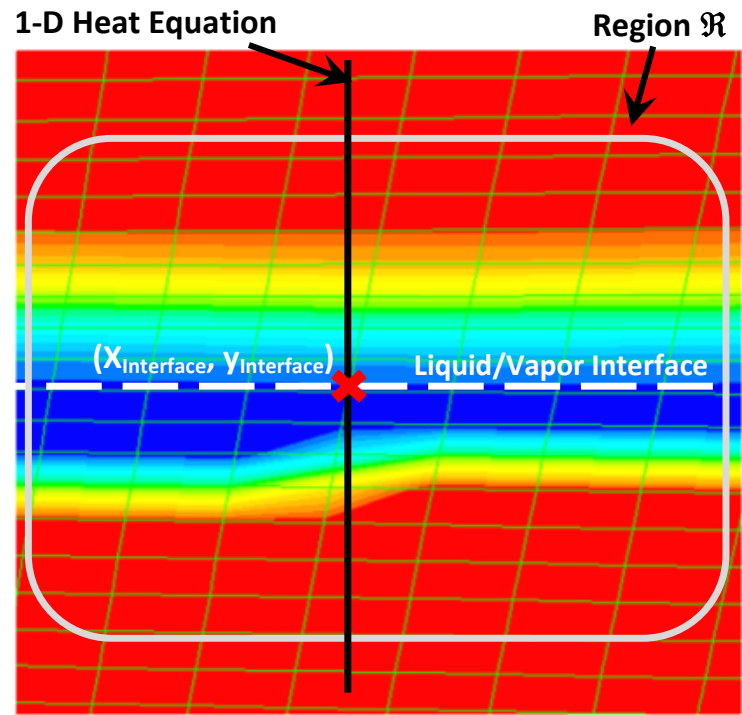

Figure 6: Subgrid model moves with the liquid/vapor interface and solves the 1-D heat equation normal to it. Four couplings exist with the fluid simulation. Mass and energy added to cells (blue) near the interface.

$\left(-k_{\text {liq }} \frac{d T}{d x}\right)_{\text {interface-liq }},\left(-k_{\text {vap }} \frac{d T}{d x}\right)_{\text {interface-vap }}$, is calculated from the subgrid model (1-D heat equation), and included as source/sink terms in the liquid/vapor phase energy equations in the fluid simulation.

In general, the momentum corresponding to interface mass transfer would also be represented with source/sink terms, but it is considered negligible in these simulations. In the EDU simulations, $\Re$ is a single, thin $(6 \mathrm{~cm}-\mathrm{a}$ few cells wide) rectangular region, centered on $x_{\text {interface }}$, covering the entire interface from tank axis to the wall. A single 1-D grid on the interface is adequate for proof of concept, but it does not capture variations along the interface.

\section{Energy and mass are conserved adaptively}

For an interface tracking scheme, the interface is not a line/surface but a set of grid cells near the interface, as in Figure 6. The number and volumes of these cells is unknown and changing - changing slowly relative to the time step. To accommodate this volume variation and get the proper mass/energy source terms, scaling factors, $\beta$ and $\chi$, are calculated in $\Re$, each time step, Eq. 15, 16.

$$
\begin{aligned}
& \dot{m}_{\text {flux }}^{\text {eff }}=\beta_{j-1} \frac{\sum_{\Re} \dot{m}_{\text {interface }} \varphi_{\text {vap }} d V}{A_{\text {interface }}^{\text {frerface }}} \quad \beta_{j}=\beta_{j-1} \frac{\dot{m}_{f l u x}}{\dot{m}_{\text {flux }}^{\text {eff }}} \text {, similarly for the liquid } \\
& \dot{q}_{\text {flux }}^{\text {eff }}=\chi_{j-1} \frac{\sum_{\Re} \dot{q}_{\text {interface }} \varphi_{\text {vap }} d V}{A_{\text {interface }}^{\text {? }}} \quad \chi_{j}=\chi_{j-1} \frac{\dot{q}_{f l u x}}{q_{\text {flux }}^{\text {eff }}}, \text { similarly for the liquid }
\end{aligned}
$$

Extensions of this scheme would include a non-uniform 1-D grid to resolve the liquid/vapor interface while capturing profile details distant from the interface.

\section{Fluid and Material Properties}

Hydrogen liquid is treated as a Boussinesq fluid, while the vapor is an ideal gas. A real gas differs from an ideal gas by up to $12 \%$ over the temperatures and pressures in the simulation, particularly near the saturation line. This must be addressed.

Constant physical properties at reference conditions, $(20.207 \mathrm{~K}, 99.224 \mathrm{kPa})$, do not adequately capture thermal conductivity, $k$, in the gas phase and in the aluminum tank walls. Linear equations in temperature accurately represent NIST data [19] for viscosity, $\mu$, and thermal conductivity, $k$, of the gas phase hydrogen. 
The saturation line conditions for hydrogen, $p_{\text {sat }}(T)$ and $\Delta H_{\text {vap }}(T)$, are represented by cubic polynomials least squares fit to NIST data [19] (Figure 7) over the interface temperature range.

NIST data [20] for 5083 aluminum is used for piecewise linear representations of the specific heat, $C$, and thermal conductivity, $k .5083$ aluminum data is indistinguishable from 2219 aluminum data for thermal conductivity, and cryogenic data is publicly available. NIST data [20] for 304 stainless steel is also used.

\section{The Experiment, Geometry, and Grid}

The cryogenic tank simulated is the EDU (or IVF 1000) rank built at Marshall Space Flight Center with preliminary testing in June 2014 and Phase A testing in September 2015. Figure 8 and Figure 9 show the tank and axisymmetric geometry. This test article (not flight

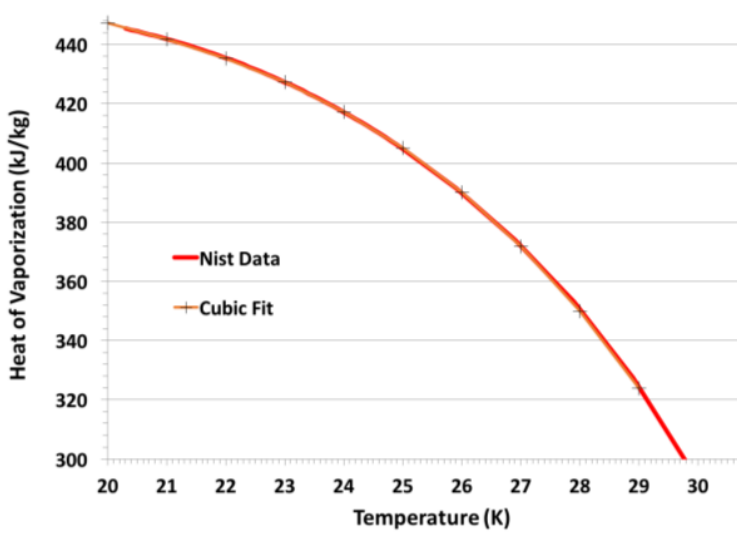

Figure 7: Latent heat, $\Delta H_{v a p}(T)$, for hydrogen is accurately represented (error $<0.1 \%$ ) by a thirdorder polynomial when used in Eq. 5. NIST Data [18].

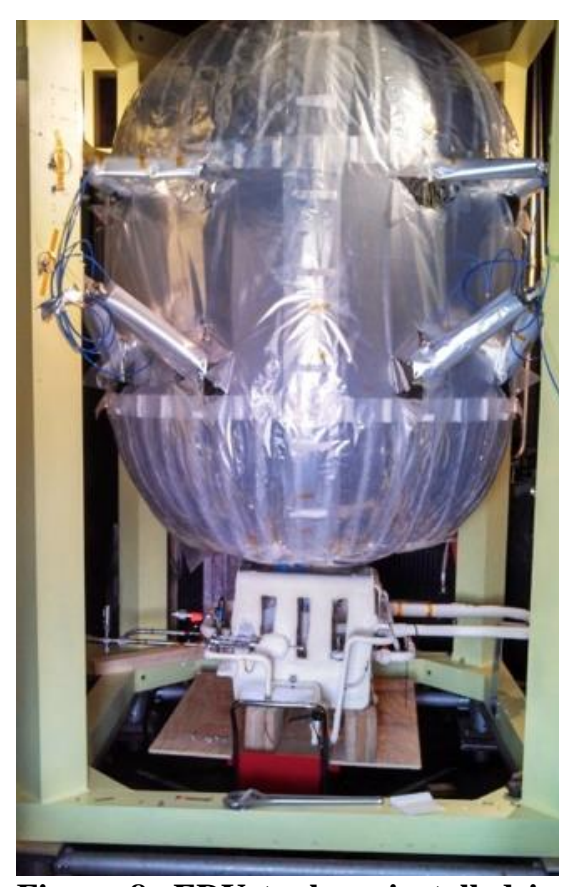

Figure 8: EDU tank as installed in TS 300. weight) was built to gain experience in tank design and construction, and to test cryogenic fluid management techniques. There is no final report to date on this tank or test, only design notes and testing data.

This tank is constructed from 2219 aluminum, insulated with 1.25 inches of SOFI, 20 layers of low density MLI and 40 layers of standard density MLI. Tank internal volume is $4.336 \mathrm{~m}^{3}$, inner diameter $1.70 \mathrm{~m}$, inner height $2.33 \mathrm{~m}$, dome ratio $\sqrt{2} / 2: 1$. The nominal wall thickness is $2.54 \mathrm{~mm}$. It was tested in vacuum conditions at $300 \mathrm{~K}$ shroud temperature and 1 -g.

\section{Geometry}

Simulation geometry comes directly from design CAD files, and many internal components have been removed. A single axisymmetric penetration is kept in the top lid, and included in the bottom lid, for unsubmerged and submerged diffusers and their 304 stainless steel supply lines. Figure 9 and Figure 10 show the supply lines

Vent (vapor) and drain (liquid) are by sink terms for mass, momentum, and energy equations implemented in UDFs and geometrically located in small, radius $10 \mathrm{~cm}$, circular regions near the tank top and bottom. Venting modifies outflow mass flow to achieve a specified maximum pressure. Drainage is implemented through an orifice plate equation with a $3.97 \mathrm{~mm}$ orifice in a $10.21 \mathrm{~mm}$ I.D. pipe, flow coefficient 0.98 , the pressure at the tank bottom, and a prescribed back pressure.

\section{Grid}

The computational domain is two-dimensional, axisymmetric, as shown in Figure 10. The baseline grid contains 37,400 cells, 2100 represent the solid walls and 35,300 the fluid. A multi-block structured grid captures variations in wall thickness. Further, an unstructured grid represents the complex geometry of the lid of the manhole access and the diffuser interior. Typical grid scale is $\sim 5 \mathrm{~cm}$ vertical resolution and $\sim 10 \mathrm{~cm}$ horizontal resolution.

In the grid and simulation, each diffuser supply line empties into the diffuser body. The actual diffuser has multiple holes that allow flow into the tank after reducing its speed and momentum - slow flow reduces mixing of the tank contents. In the grid, the diffuser must be axisymmetric and it is modelled as an equivalent slot as shown in Figure 10 on the right. 


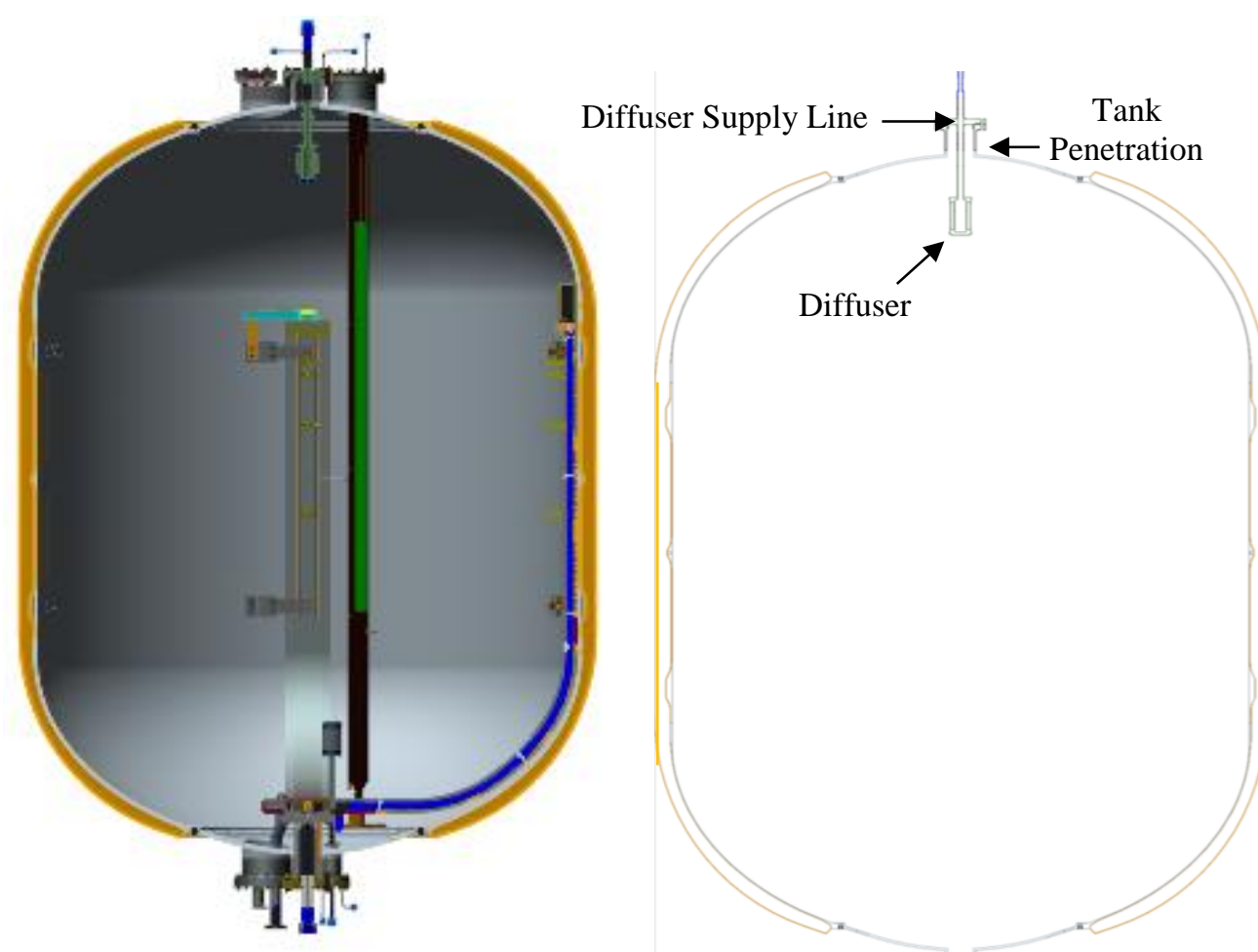

Figure 9: Cross sectional view of eCryo EDU tank (left), with axisymmetric view (right). The simulations are axisymmetric, and many internal components have been removed (right).
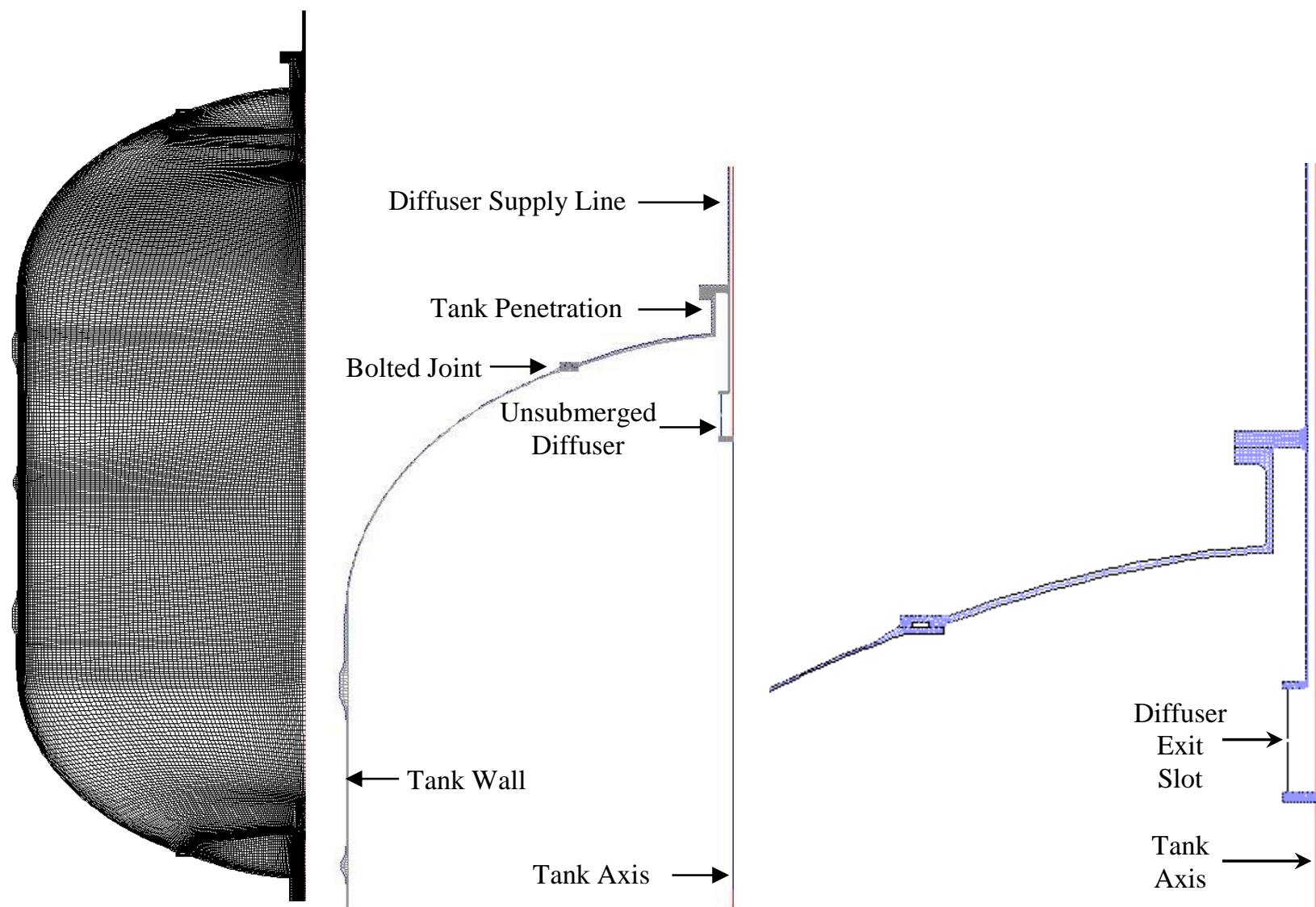

Figure 10: Axisymmetric fluid+walls grid (left), walls only(center), including unsubmerged diffuser, tank wall with bolted joint, and tank penetration for diffuser supply line, closeup (right). Top and bottom tank penetrations are midline symmetric. SOFI and MLI are part of the tank wall boundary condition-not the grid. 


\section{Comparison with Experimental Results}

One test within Phase A EDU testing was chosen for simulation. This experimental data is a good test of the subgrid interface model from Section IV. The interface must be tracked for over $140 \mathrm{~s}$, and the dramatic pressure increase results in a large temperature jump with thermal layers on both sides of the interface. In a series of developmental simulations, the interface subgrid model has been extensively exercised, tested, and a stable numerical scheme is apparent.

Figure 11 shows the test data for test HT-15, 16 on day 3 of Phase A testing. The tank was filled with LH2 to the $90 \%$ fill level, with GH2 in the ullage, and left to settle. GH2 pressurant gas at $298 \mathrm{~K}$ was introduced through the unsubmerged diffuser supply line. Although the drainage line valve was open, there was a limited change in interface height-- 1\% fill level reduction.

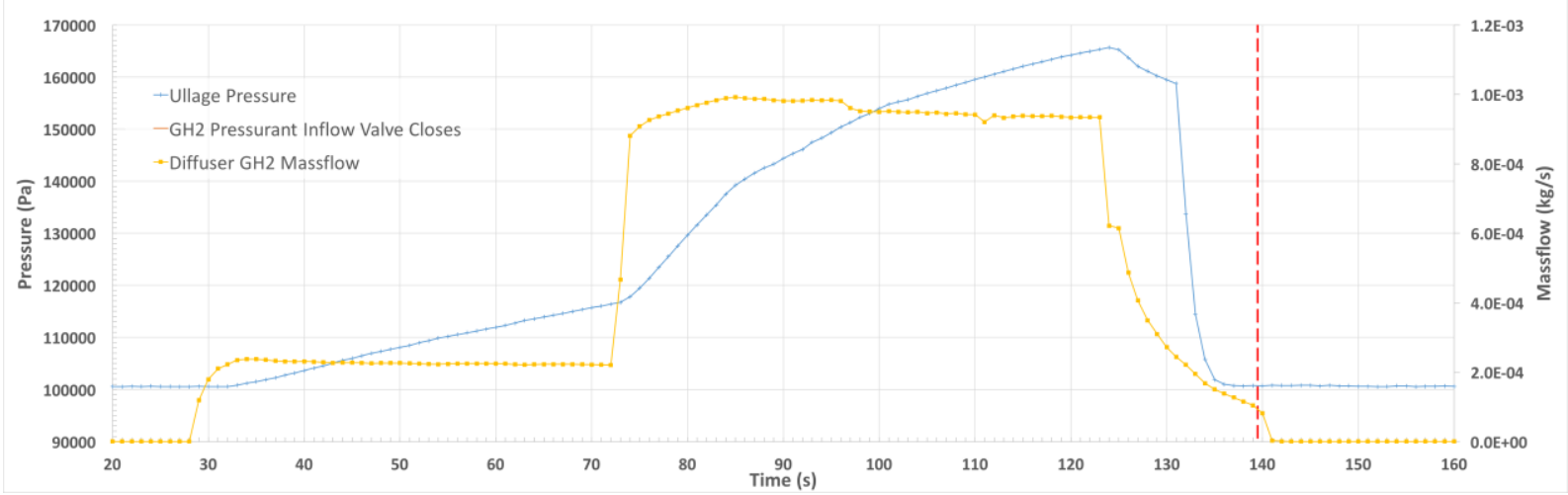

Figure 11: Experimental measurements of ullage pressure rise in tank, inflowing diffuser GH2 pressurant, and pressurant inflow valve closing time.

Figure 12 shows velocity vectors and temperature in and near the diffuser, tank penetration, and supply lines. The heat introduced through the pressurant flow is substantial and the supply line conducts heat into the tank. The grid for the inflow line resolves the momentum and thermal boundary layers and predicts heat flow into the supply line. There is hot gas in the upper part of the tank, as inflowing pressurant is buoyantly driven upwards after exiting from the diffuser.
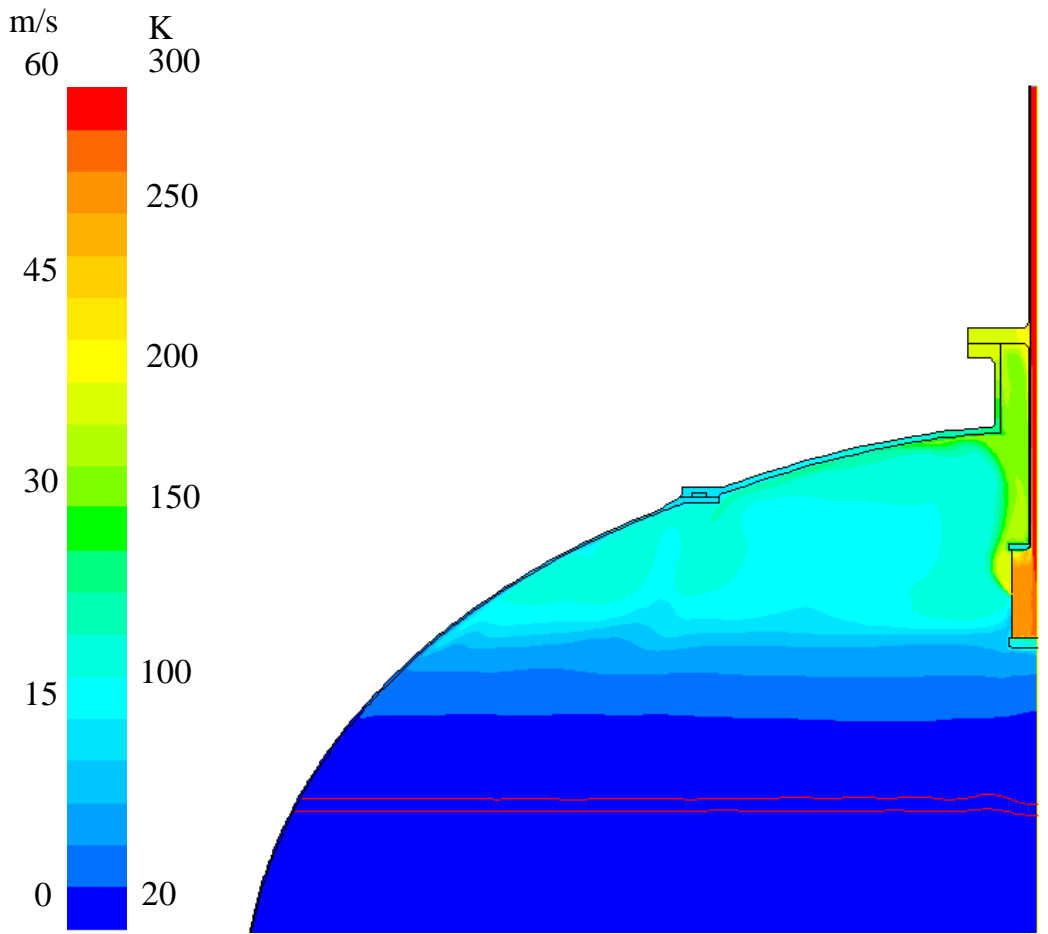

Figure 12: Velocity vectors in the unsubmerged diffuser (left) and temperature distribution through the top of the tank (right). The diffuser reduces the momentum of the pressurant. 


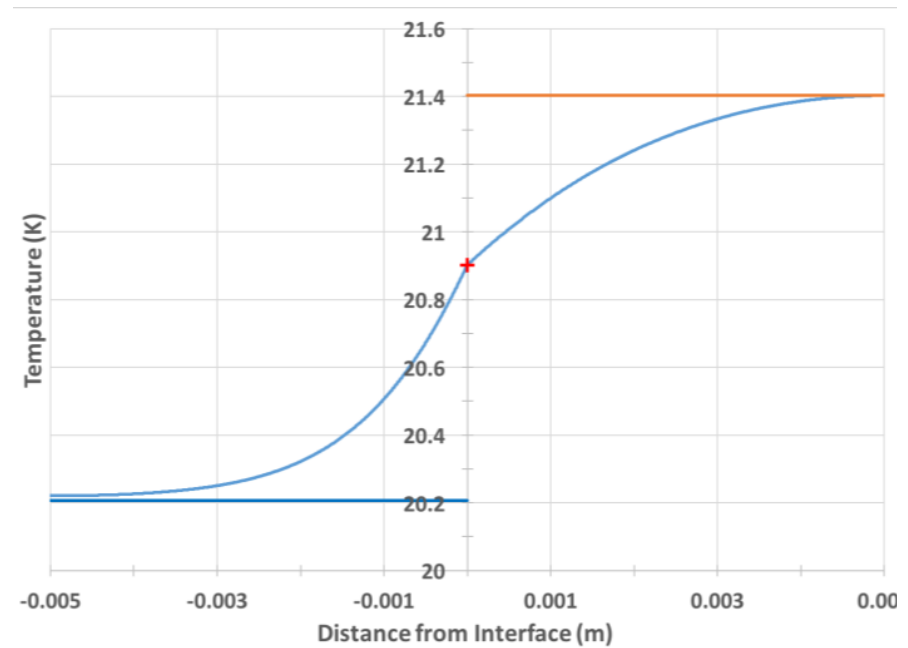

Figure 13: Temperature profile through the liquid/vapor interface as measured in the subgrid model at $t=87.23 \mathrm{~s}$. Red plus indicates the interface position and temperature.

Figure 13 shows the temperature profile through the liquid/vapor interface at $\mathrm{t}=87.23 \mathrm{~s}$ as calculated by the subgrid model. The temperature jump is greater than one degree $\mathrm{K}$, and the interface temperature-where the HKS equation is applied-is a point on the temperature profile. The heat flux from the vapor to the interface is $4 \mathrm{~W} / \mathrm{m}^{2}$, condensation at the interface adds almost $53 \mathrm{~W} / \mathrm{m}^{2}$, and the liquid absorbs $54 \mathrm{~W} / \mathrm{m}^{2}$ from the interface. The balance is heating at the interface. The condensation mass flux is $1 \times 10^{-4} \mathrm{~kg} / \mathrm{m}^{2}$-s.

Figure 14 compares the calculated pressure transient with the experimental data. For most of the experiment, the loss of vapor to condensation is smaller than the pressurant inflow; the ratio of pressurant mass flow added to $\mathrm{GH} 2$ condensed is near 3:-1. But, beginning at $123 \mathrm{~s}$ and until $131 \mathrm{~s}$, the pressurant massflow drops by nearly an order of magnitude until a relief valve is opened. Condensation mass flux has a more significant effect on pressure in this region. As such it is a better test of the interface model and predicted condensation rate.

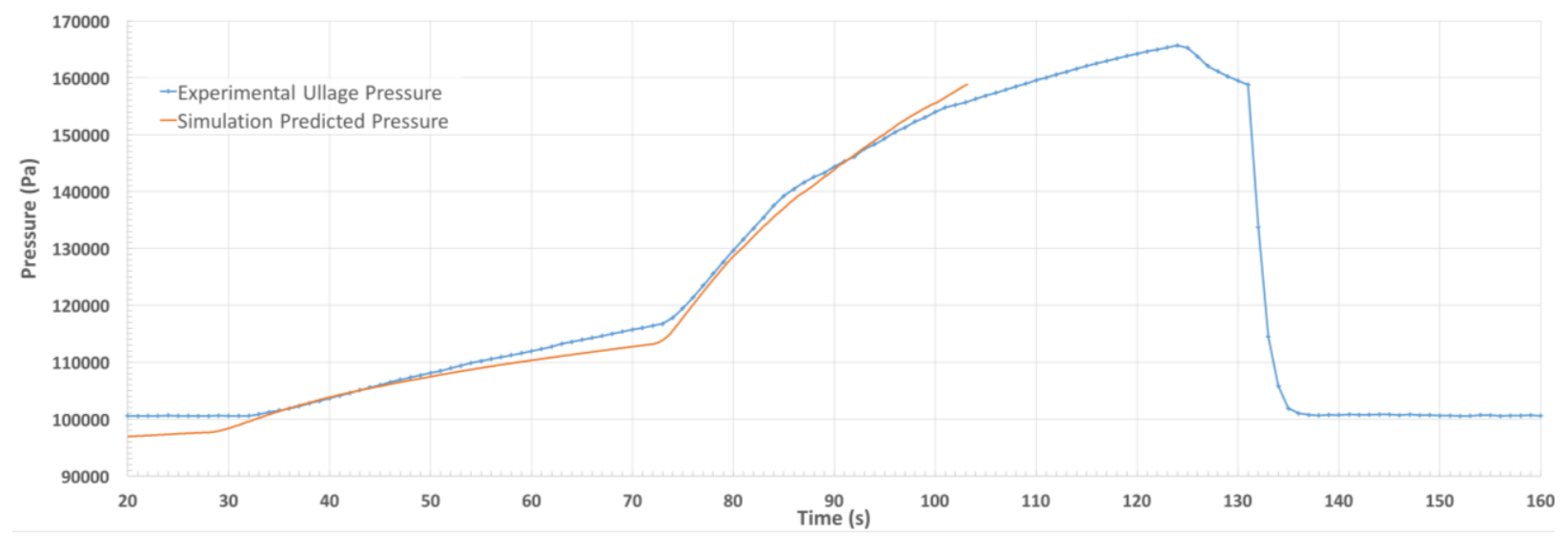

Figure 14: Comparison of computational pressure predictions and experimental measurements. Between 123 and $131 \mathrm{~s}$, pressurant inflow drops, condensation mass flux becomes more significant in the pressure profile, and a better test of predicted condensation rate.

\section{Conclusion}

The analysis, algorithms, and results of this paper provide proof of concept for a numerical method for interfacial mass transfer. Although applied to hydrogen, the approach should apply to other fluids. The use of an accommodation coefficient of 1.0 in these calculations is consistent with theory and experiments, and adds confidence to this approach. Further development of the methods is needed, including extensions to curved interfaces with multiple interface sections, $\mathfrak{R}$. Several problems should be reconsidered; with slosh, turbulent thermal conductivity will increase heat conduction through the thermal layers to the interface, accelerate interfacial mass transfer, and possibly explain pressure collapse in orbit tanks.

\section{Acknowledgments}

The authors thank Andrè LeClair, Jason Hartwig, Wesley Johnson for helpful discussions. Aimee Dechant, Monica Guzik, Dale Diedrick, and Andy Hissam helped locate the EDU CAD data. John Ibrahim and Valerio Viti 
from Fluent helped with simulation questions. This work was supported under the NASA Space Technology Mission Directorate's Technology Demonstration Missions Program under the Evolvable Cryogenics Project.

\section{References}

[1] O. Kartuzova and M. Kassemi, "Modeling Interfacial Turbulent Heat Transfer during Ventless Pressurization of a Large Scale Cryogenic Storage Tank in Microgravity," in AIAA, 2011.

[2] M. E. M. Stewart and J. P. Moder, "Self-Pressurization of a Flightweight, Liquid Hydrogen Tank: Simulation and Comparison with Experiments," in AIAA Propulsion and Energy Conference, AIAA-2016-4674 Salt Lake City, 2016.

[3] H. W. Liepmann and A. Roshko, Elements of Gasdynamics, New York: John Wiley \& Sons, 1958.

[4] M. E. M. Stewart and J. P. Moder, "Comparison of Computation Results with a Low-g, Nitrogen Slosh and Boiling Experiment," in AIAA Propulsion and Energy Conference, AIAA 2015-3854, Orlando, July 2015.

[5] B. J. Mason, The Physics of Clouds, Oxford: Oxford University Press, 1971.

[6] S. Popov, A. Melling, F. Durst and C. A. Ward, "Apparatus for investigation of evaporation at free liquid-vapur interfaces," International Journal of Heat and Mass Transfer, vol. 48, pp. 2299-2309, 2005.

[7] V. K. Badam, V. Kumar, F. Durst and K. Danov, "Experimental and theoretical investigations on interfacial temperature jumps during evaporation," Experimental Thermal and Fluid Science, vol. 32, pp. 276-292, 2007.

[8] M. S. Raju, "LSPRAY-V: A Lagrangian Spray Module," NASA/CR--2015-218918, 2015.

[9] M. S. Raju and W. A. Sirignano, "Interaction between two vaporizing droplets in an intermediate Reynolds number flow," Physics of Fluids A, vol. 2, pp. 1780-1796, Oct. 1990.

[10] J. B. Mehl and M. R. Moldover, "Precondensation phenomena in acoustic measurements," J. Chem. Phys., vol. 77, no. 1, pp. 455-465, July 1982.

[11] M. B. Ewing, M. L. McGlashan and J. P. M. Trusler, "The Temperature-Jump Effect and the Theory of the Thermal Boundary Layer for a Spherical Resonator. Speeds of Sounds in Argon at 271.16K," Metrologia, vol. 22, pp. 93-102, 1986.

[12] R. W. Schrage, A Theoretical Study of Interphase Mass Transfer, Columbia University Press, 1953.

[13] J. Vieceli, M. Roeselova and D. J. Tobias, "Accommodation coefficients for water vapor at the air/water interface," Chemical Physics Letters, vol. 393, pp. 249-255, 2004.

[14] M. Matsumoto, K. Yasuoka and Y. Kataoka, "Evaporation and condensation at a liquid surface. II. Methanol," J. Chem. Phys., vol. 101, no. 9, pp. 7912-7917, Nov. 1994.

[15] K. Yasuoka, M. Matsumoto and Y. Kataoka, "Evaporation and condensation at a liquid surface. I. Argon," $J$. Chem. Phys. , vol. 101, no. 9, pp. 7904-7911, Nov. 1994.

[16] W. H. Press, B. P. Flannery, S. A. Teukolsky and W. T. Vetterling, Numerical Recipes in C: The Art of Scientific Computing, New York: Cambridge Univ. Press, 1988.

[17] ANSYS, ANSYS Fluent Software, Ver. 16.0, Canonsburg, PA: ANSYS, Inc., Dec 2014.

[18] ANSYS, ANSYS Fluent Theory Guide, Release 17.2, Canonsburg, PA: Ansys, Inc., Aug 2016.

[19] NIST, "Thermophysical Properties of Fluid Systems," National Institute for Standards and Technology, 2011. [Online]. Available: http://webbook.nist.gov/chemistry/fluid. [Accessed January 2015].

[20] NIST, "Material Measurement Laboratory: Cryogenics Technologies Group," [Online]. Available: http://cryogenics.nist.gov/MPropsMAY/materialproperties.htm. [Accessed 2015 April]. 